\title{
Health related quality of life and psychological wellbeing in patients with dilated cardiomyopathy
}

\author{
A Steptoe, A Mohabir, N G Mahon, W J McKenna
}

\begin{abstract}
Objective-To assess the health related quality of life and psychological wellbeing of patients with dilated cardiomyopathy, and relate these to clinical variables and psychological adjustment. Design-Postal questionnaire survey of 99 adult patients with dilated cardiomyopathy, selected at random from a larger database (60.6\% response rate). Assessments included the short form 36 (SF-36) health survey, the hospital anxiety and depression scales, the sleep problems index, and a measure of psychological adjustment to cardiomyopathy.

Results-Patients with dilated cardiomyopathy reported significant impairments in physical functioning, role limitations owing to physical and emotional problems, social functioning, mental health, perceptions of general health, sleep, and vitality. Anxiety and depression levels were higher than in population samples. Impairment in several domains of quality of life was associated with low shortening fraction, high left ventricular end diastolic diameter, and the presence of heart failure and mitral regurgitation. Patients with familial cardiomyopathy had less impairment in quality of life than those with non-familial disease. Psychological adjustment scores were associated with several aspects of quality of life and emotional wellbeing. In multivariate analysis, demographic and clinical variables accounted for $0.1-40.7 \%$ of the variance in different domains of quality of life, and psychological adjustment scores accounted for an additional $0.5-22.4 \%$ of variance.

Conclusions-Patients with dilated cardiomyopathy experience pronounced restrictions in quality of life and psychological wellbeing. These limitations are only partly accounted for by symptoms and the severity of underlying disease. Patients may benefit from efforts to improve psychological adjustment to the condition.
\end{abstract}

(Heart 2000;83:645-650)

Keywords: dilated cardiomyopathy; quality of life; adjustment; wellbeing

Dilated cardiomyopathy is a multifactorial syndrome characterised by cardiac dilatation and systolic dysfunction. ${ }^{12}$ It can lead to substantial limitations in daily activities, while the poor prognosis may result in emotional distress among patients and their families. There is little systemic evidence, however, concerning health related quality of life and psychological wellbeing in this disorder. The aim of the present study was to assess quality of life and psychological state in patients with dilated cardiomyopathy using standardised measures, so that comparisons could be made with reference populations and other cardiovascular disease groups. We used a protocol similar to that previously administered to patients with hypertrophic cardiomyopathy in the same clinic, enabling direct comparisons to be made. ${ }^{3}$ We previously found that a short measure of psychological adjustment to cardiomyopathy was associated with several aspects of quality of life, so this was included in the present analysis. Patients with familial and non-familial dilated cardiomyopathy were compared, since knowledge of a hereditary component might have adverse effects on wellbeing. In addition, the relation of quality of life and psychological wellbeing with symptoms and clinical indices of disease (extent of fraction shortening, left ventricular end diastolic diameter, exercise capacity, and the presence of heart failure, cardiac arrhythmia, and mitral regurgitation) was evaluated.

\section{Patients and methods}

One hundred and six patients who had attended a dilated cardiomyopathy clinic at St George's Hospital, London, over the past 12 months were randomly selected from the database. Dilated cardiomyopathy was defined by characteristic clinical, echocardiographic, and haemodynamic features. ${ }^{4}$ Each patient was sent an explanatory letter and a set of questionnaires, with a reply paid envelope. Complete questionnaires were returned by 60 patients; seven had died or had moved house and 39 failed to respond, giving a response rate of $60.6 \%$. Comparisons between responders and non-responders on demographic variables, New York Heart Association (NYHA) grading, shortening fraction, and presence or absence of cardiac arrhythmia and mitral regurgitation showed no significant differences between groups.

\section{MEASURES}

Quality of life

Quality of life was assessed using the short form 36 health survey (SF-36), a multidimensional measure that has been widely used in quality of life studies. ${ }^{5}$ The measure has been used with a variety of clinical groups in the $\mathrm{UK},{ }^{6}$ and normative data for the general population are available. ${ }^{78}$ Eight dimensions of functioning are assessed with the SF-36: physical functioning or activities of daily living; role limitations owing to physical problems; role 
limitations resulting from emotional problems; social functioning; mental health; general perceptions of health; vitality or energy; and bodily pain. Scores were coded and transformed to a scale where $0=$ worst possible health and $100=$ best possible health.

Quality of sleep

Sleep quality was assessed using the six item sleep problems index developed in the medical outcomes study (MOS). ${ }^{9}$ Individual items assessed difficulty falling asleep, maintenance of sleep, and tiredness during the day. Ratings were summed to range up to a maximum of 36, with higher scores indicating better sleep function. The internal consistency (Cronbach $\alpha$ ) of the measure in the present study was 0.84 .

\section{Psychological wellbeing}

Anxiety and depression were assessed with the hospital anxiety and depression (HAD) scale, a 14 item questionnaire designed to assess emotional distress with items uncontaminated by somatic symptoms. ${ }^{10}$ The measure has been used in more than 200 published studies in different medical settings, and has been validated against psychiatric interviews. ${ }^{11}$ The questions address the patient's emotions over the past few weeks, with seven items related to depression (for example, "I still enjoy the things I used to enjoy", reverse scored), and seven related to anxiety (for example, "Worrying thoughts go through my mind"), each of which is rated on a four point scale. Scores can range from 0-21, with higher scores reflecting greater degrees of anxiety and depression. Scores of eight or more are suggestive of borderline emotional disorder, while scores of 11 or more are indicative of probable emotional disorder. Cronbach $\alpha$ scores were 0.81 for the anxiety and 0.89 for the depression subscales in this sample.

\section{Psychological adjustment to cardiomyopathy}

Adjustment was assessed with five items developed in a previous study of hypertrophic cardiomyopathy. ${ }^{3}$ Each item (for example, "A person with cardiomyopathy can lead a fully satisfying life", and "I worry that a diagnosis of cardiomyopathy is a death sentence", reverse scored) was scored on a five point rating scale from "strongly disagree" to "strongly agree", and ratings were summed to produce a total score with a potential range of 5-25. Higher scores indicate better adjustment to cardiomyopathy. The internal consistency of the scale in this study was good (Cronbach $\alpha=0.83$ ). Patients were divided into those with poor and good adjustment based on a criterion of 13.5 on this scale.

DEMOGRAPHIC AND CLINICAL DATA

Age, sex, marital and employment status, NYHA class, left ventricular shortening fraction, end diastolic diameter, exercise capacity, and the presence or absence of cardiac arrhythmia and mitral regurgitation were abstracted from clinical records. Familial dilated cardiomyopathy was defined as having one or more relatives with established disease or left ventricular enlargement. All patients had a shortening fraction $<30 \%$, but for the purposes of analysis they were subdivided into those with lower $(\leqslant 18 \%)$ and higher $(>18 \%)$ shortening fraction. Comparisons were made between patients with left ventricular end diastolic diameters greater or less than $6.5 \mathrm{~cm}$. Peak oxygen consumption $\left(\mathrm{Vo}_{2}\right)$ during exercise testing was converted to per cent predicted maximal $\mathrm{VO}_{2}$ adjusted for age, body surface area, and sex, and comparison was made between patients whose peak $\mathrm{VO}_{2}$ was above or below $80 \%$. Individuals were excluded from the analysis of exercise capacity if the respiratory quotient during exercise testing was $<1$. Patients were managed according to conventional algorithms for the prevention of complications and treatment of symptoms of heart failure. In brief, non-invasive risk factor stratification for arrhythmia, embolic and sudden death risk was performed, and the threshold was low for the introduction of amiodarone for prevention of arrhythmia, and anticoagulants for prevention of emboli. Symptomatic treatment aimed to achieve the highest tolerated dose of either an angiotensin converting enzyme inhibitor or an angiotensin II antagonist. The use of diuretics was limited to those patients with congestive symptoms.

\section{STATISTICAL METHODS}

Comparisons between the hypertrophic cardiomyopathy patients and previously published findings for quality of life and psychological wellbeing were carried out using $t$ tests. The associations of quality of life and psychological wellbeing with clinical features were evaluated by analysis of covariance, using sex, age, and duration of illness as covariates. Multivariate analysis of factors associated with quality of life and wellbeing was carried out using multiple regression methods.

\section{Results}

The characteristics of the patients are summarised in table 1 . The population was predominantly male with an age range of 16-77 years (mean 47.6 years); the interval since diagnosis ranged from 1-60 months. Nearly half the patients had familial cardiomyopathy. Cardiac arrhythmia was present in about one third, while $31.6 \%$ had moderate or severe mitral regurgitation. Forty per cent of patients had heart failure according to their NYHA grade, and comorbidity was present in $20.7 \%$.

Mean scores on the quality of life and psychological wellbeing scales are summarised in table 2. This sample of patients with dilated cardiomyopathy showed major limitations in all dimensions of the SF-36 except for bodily pain. In each case, quality of life scores were significantly lower than those of the UK general population $(p<0.025$ to $p<0.0001) .^{8}$ Dilated cardiomyopathy patients evidently experience restrictions in physical functioning, fulfilment of roles in life, social functioning, mental health and vitality, and perceive their general health to be poor.

The comparison with the sample of hypertrophic cardiomyopathy patients from St George's Hospital studied by Cox and 
Table 1 Demographic and clinical features of the sample

\begin{tabular}{lc}
\hline Sex & $40 \mathrm{M}, 20 \mathrm{~F}$ \\
Age & $47.6(14.4)$ \\
Months since diagnosis & $42.6(47.0)$ \\
Marital status & \\
$\quad$ Single & 23.7 \\
$\quad$ Married & 64.4 \\
$\quad$ Divorced/widowed & 11.9 \\
Occupation & \\
$\quad$ Paid employment & 51.6 \\
Retired (age) & 10.0 \\
Retired (illness) & 20.0 \\
Unable to work (illness) & 11.7 \\
Not in paid employment & 6.7 \\
Familial cardiomyopathy & 42.6 \\
Comorbidity & 20.7 \\
Left ventricular shortening fraction & $18.1(5.3)$ \\
Left ventricular end diastolic diameter (cm) & $5.45(0.93)$ \\
Cardiac arrhythmia & 30.2 \\
Mitral regurgitation (moderate or severe) & 31.6 \\
Per cent of predicted peak Vo ${ }_{2}$ & $75.7(26.5)$ \\
NYHA classification & \\
$\quad$ Grade I & 60.0 \\
Grade II & 32.7 \\
Grade III or IV & 7.3 \\
\hline
\end{tabular}

Values are mean (SD) or \%.

colleagues $^{3}$ showed only two differences on the SF-36. The dilated cardiomyopathy group reported significantly greater restrictions in social functioning (controlling for age, sex, and duration of illness, $p<0.001$ ), while the hypertrophic patients experienced higher levels of pain $(\mathrm{p}<0.003)$. Comparison was also made with 144 patients with serious cardiac conditions, including survivors of acute myocardial infarction with severe angina, hypertensive patients with severe congestive heart failure, patients with congestive heart failure reporting oedema, orthopnoea or dyspnoea on exertion, and diabetic patients with complications, included in MOS. ${ }^{12}$ There were no differences between the dilated cardiomyopathy and this sample of patients with serious cardiac conditions in terms of physical functioning, physical role limitations, emotional role limitations, vitality, or general perceptions of health. The dilated cardiomyopathy group were, however, more limited in terms of social functioning $(\mathrm{p}<0.001)$ and had poorer mental health $(p<0.0001)$, while the serious cardiac condition group experience more pain $(\mathrm{p}<0.001)$.

Table 2 Quality of life and psychological wellbeing of patients with dilated cardiomyopathy

\begin{tabular}{llll}
\hline & $\begin{array}{l}\text { Dilated } \\
\text { cardiomyopathy }\end{array}$ & $\begin{array}{l}\text { Hypertrophic } \\
\text { cardiomyopathy }^{3}\end{array}$ & $\begin{array}{l}\text { General } \\
\text { population }^{8}\end{array}$ \\
\hline $\mathrm{n}$ & 60 & 137 & $8883-9219$ \\
Physical functioning (SF-36) & $58.3(24.6)^{\star}$ & $60.9(25.3)$ & $88.4(18.0)^{\star}$ \\
Physical role limitations (SF-36) & $42.9(38.8)^{\star}$ & $55.9(42.9)$ & $85.8(29.9)^{\star}$ \\
Emotional role limitations (SF-36) & $67.2(41.4)^{\star}$ & $64.2(40.7)$ & $82.9(31.8)^{\star}$ \\
Social functioning (SF-36) & $57.0(8.1)^{\star} \dagger$ & $70.2(26.3) \dagger$ & $88.0(19.6)^{\star}$ \\
Mental health (SF-36) & $68.9(21.1)^{\star}$ & $65.6(21.2)$ & $73.8(17.2)^{\star}$ \\
General health perceptions (SF-36) & $47.0(18.7)^{\star}$ & $47.2(24.4)$ & $73.5(19.9)^{\star}$ \\
Vitality (SF-36) & $46.1(22.1)^{\star}$ & $43.6(24.2)$ & $61.1(19.7)^{\star}$ \\
Pain (SF-36) & $77.6(19.8) \dagger$ & $66.1(27.2) \dagger$ & $81.5(21.7)^{\star}$ \\
MOS sample ${ }^{9}(\mathrm{n}=3053)$ & & & \\
$\quad$ Sleep (MOS) & $23.2(6.6)^{\star}$ & & $28.3(18.2)^{\star}$ \\
General population ${ }^{13}(\mathrm{n}=624)$ & & & \\
$\quad$ Anxiety (HAD) & $8.10(3.9)^{\star}$ & $8.23(4.3)$ & $4.55(3.7)^{\star}$ \\
$\quad$ Depression (HAD) & $5.17(4.3)^{\star}$ & $5.29(3.6)$ & $3.98(3.5)^{\star}$ \\
\hline
\end{tabular}

Values are mean (SD).

$389{ }^{13}$ References of comparison samples; * significant difference between dilated cardiomyopathy and general population comparison samples, $\mathrm{p}<0.025$; †significant difference between dilated cardiomyopathy and hypertrophic cardiomyopathy, $\mathrm{p}<0.003$.
Scores on the sleep problems index were significantly lower than those reported by the MOS sample $(p<0.05)$, indicating that sleep is impaired among patients with dilated cardiomyopathy. ${ }^{9}$ No norms for the HAD anxiety and depression scales have been published in the UK, so comparison was made with a Swedish population sample. ${ }^{13}$ Significantly higher levels of both anxiety and depression were found in dilated cardiomyopathy $(p<0.001)$. Fifty two per cent of patients fulfilled the criteria for a possible case of anxiety disorder, and $22 \%$ scored above the criterion for a possible case of clinical depression. These levels are substantially higher than those reported in the population samples. ${ }^{11}$ There was no significant difference between the dilated and hypertrophic cardiomyopathy patients in anxiety or depression. Data have recently been published describing HAD scores in 385 mixed medical inpatients. ${ }^{14}$ Anxiety scores were significantly higher in dilated cardiomyopathy compared with this sample (mean (SD) 6.9 (3.9), p < 0.05), but depression levels were comparable (mean 5.5 (3.9)).

ASSOCIATIONS WITH DEMOGRAPHIC AND CLINICAL FEATURES

Quality of life and psychological wellbeing were not significantly associated with the duration of illness, as indexed by the interval since diagnosis. Older patients reported significantly greater vitality and perceived their general health to be better than did younger patients $(\mathrm{p}<0.01)$. Comparisons between male and female patients were carried out after adjustment for age and duration of illness. Female patients showed significantly poorer quality of life than male patients in terms of physical function $(p<0.01)$, perceptions of general health $(\mathrm{p}<0.025)$, and bodily pain $(\mathrm{p}<0.025)$, but men and women did not differ in psychological wellbeing.

The association of quality of life and psychological wellbeing with clinical features were analysed after controlling for sex, age, and duration of illness. Results related to the measures of systolic and diastolic myocardial function are shown in table 3. Patients with lower shortening fractions had poorer quality of life in terms of physical function and mental health than did those with better left ventricular function $(p<0.05)$. Eight individuals had left ventricular end diastolic diameters greater than $6.5 \mathrm{~cm}$. These patients were more impaired than the remainder in physical functioning, physical role limitations, general health perceptions, and sleep quality $(p<0.05)$. No association between quality of life, sleep, or emotional state and the presence of cardiac arrhythmia could be detected. There were also no significant relations with low per cent predicted peak $\mathrm{VO}_{2}$. The presence of familial cardiomyopathy was related to quality of life, but not as anticipated. As can be seen in table 4, patients with familial cardiomyopathy reported better physical function, fewer role limitations because of emotional factors, and better sleep quality than the remainder $(\mathrm{p}<0.05)$. Mitral regurgitation was also 
Table 3 Quality of life and psychological wellbeing in relation to shortening fraction and end diastolic diameter (mean (SD) adjusted for age, sex, and duration of illness)

\begin{tabular}{|c|c|c|c|c|}
\hline \multirow[b]{3}{*}{ Physical functioning (SF-36) } & \multicolumn{2}{|c|}{ Shortening fraction } & \multicolumn{2}{|c|}{ End diastolic diameter } \\
\hline & $\leqslant 18.0 \%$ & $>18.0 \%$ & $\leqslant 6.5 \mathrm{~cm}$ & $>6.5 \mathrm{~cm}$ \\
\hline & $53.0 \quad(25.3)$ & $65.0(22.6)^{\star}$ & $63.2(23.0)$ & $43.8(29.2) \dagger$ \\
\hline Physical role limitations (SF-36) & $38.2(38.1)$ & $48.9(39.6)$ & $48.8 \quad(38.6)$ & $18.4(29.1) \dagger$ \\
\hline Emotional role limitations (SF-36) & $67.3(38.8)$ & $69.4 \quad(43.3)$ & 72.7 (38.5) & $50.0(47.1)$ \\
\hline Social functioning (SF-36) & $57.6(9.1)$ & $56.6(7.1)$ & $57.5(8.0)$ & $54.2(9.3)$ \\
\hline Mental health (SF-36) & $63.7 \quad(24.2)$ & $74.6(16.6)^{\star}$ & $(18.3)$ & $57.2(28.9)$ \\
\hline General health perceptions (SF-36) & $45.6 \quad(20.7)$ & $48.5 \quad(17.1)$ & $49.4 \quad(18.9)$ & $36.2(13.0)+$ \\
\hline Vitality (SF-36) & $43.3(23.3)$ & $49.3(21.5)$ & $47.7 \quad(22.1)$ & $40.0(20.8)$ \\
\hline Pain $(S F-36)$ & $76.3(18.9)$ & $80.7 \quad(18.5)$ & $80.7 \quad(17.5)$ & $69.5 \quad(23.7)$ \\
\hline Sleep (MOS) & $22.5(6.9)$ & $24.4(6.1)$ & $24.3(6.4)$ & $18.9(5.0) \dagger$ \\
\hline Anxiety (HAD) & $8.54(4.6)$ & $7.27(2.9)$ & $7.58(3.7)$ & $9.52(4.3)$ \\
\hline Depression (HAD) & $6.00(4.5)$ & $4.03(3.0)$ & $4.49(3.8)$ & $7.11(5.3)$ \\
\hline
\end{tabular}

$\star$ Difference between higher and lower shortening fraction groups, $\mathrm{p}<0.05$; †difference between end diastolic diameter groups, $\mathrm{p}<$ 0.05 .

Table 4 Quality of life and psychological wellbeing in relation to family history and mitral regurgitation (mean (SD) adjusted for age, sex, and duration of illness)

\begin{tabular}{|c|c|c|c|c|}
\hline \multirow[b]{3}{*}{ Physical functioning (SF-36) } & \multicolumn{2}{|c|}{ Familial cardiomyopathy } & \multicolumn{2}{|c|}{ Mitral regurgitation } \\
\hline & Present & Absent & None/mild & Moderate/severe \\
\hline & $68.6(21.0)$ & $55.2(25.4)^{\star}$ & $60.9 \quad(23.6)$ & $59.2 \quad(26.3)$ \\
\hline Physical role limitations (SF-36) & $58.7 \quad(36.2)$ & $37.1 \quad(36.2)$ & 49.7 (39.7) & 35.1 (36.6) \\
\hline Emotional role limitations (SF-36) & $88.5 \quad(42.7)$ & $59.1(42.7)^{\star}$ & $74.0 \quad(38.8)$ & $65.3(41.2)$ \\
\hline Social functioning (SF-36) & $58.7(7.3)$ & $55.7(8.2)$ & $59.1(7.2)$ & $53.9(9.3) \dagger$ \\
\hline Mental health (SF-36) & $75.5(15.8)$ & $71.4 \quad(15.8)$ & $73.8 \quad(20.2)$ & $63.4(19.6)$ \\
\hline General health perceptions (SF-36) & $51.2(18.0)$ & $45.8 \quad(17.1)$ & $49.1 \quad(18.7)$ & $45.9 \quad(19.2)$ \\
\hline Vitality (SF-36) & $53.1 \quad(20.7)$ & 45.8 (19.3) & $51.4(23.6)$ & $38.8(15.5) \dagger$ \\
\hline Pain $(\mathrm{SF}-36)$ & $82.0(15.4)$ & $79.1 \quad(18.7)$ & $80.2(18.8)$ & $76.2(18.6)$ \\
\hline Sleep (MOS) & $25.8(5.7)$ & $22.1(6.3)^{\star}$ & $24.6(6.6)$ & $21.6(6.0)$ \\
\hline Anxiety (HAD) & $6.88(2.9)$ & $7.80(3.9)$ & $7.54(4.3)$ & $8.21(2.3)$ \\
\hline Depression (HAD) & $3.70(3.7)$ & $4.82(3.7)$ & $4.44(4.5)$ & $5.70(3.3)$ \\
\hline
\end{tabular}

$\star$ Difference between positive and negative family history groups, $\mathrm{p}<0.05$; $†$ difference between mitral regurgitation groups, $\mathrm{p}<0.05$.

relevant, and was associated with poorer social functioning and reduced levels of vitality.

The comparison between the $40 \%$ of patients in heart failure (NYHA grades II-IV) and the remainder revealed three differences. Mean scores on the scale of activities of daily living (physical functioning) were significantly lower in the heart failure group (35.4 $v 47.3$, $\mathrm{p}<0.025)$, and their ratings of energy or vitality were also reduced $(37.0 v 51.4, \mathrm{p}<0.025)$. The heart failure group also experienced greater bodily pain than patients without heart failure (69.7 v81.7, $\mathrm{p}<0.025)$.

\section{ADJUSTMENT AND QUALITY OF LIFE}

The comparison of patients with poor and good adjustment scores on the adjustment to cardiomyopathy scale revealed differences in several aspects of quality of life (table 5), after controlling statistically for age, sex, and duration of illness. Patients who were poorly

Table 5 Quality of life and psychological wellbeing in relation to psychological adjustment scores (mean (SD) adjusted for age, sex, and duration of illness)

\begin{tabular}{lcccc}
\hline & \multicolumn{2}{l}{ Adjustment } & \\
\cline { 2 - 3 } & \multicolumn{2}{l}{ Poor $(<13.5)$} & Good $(>13.5)$ & Significance \\
\hline Physical functioning (SF-36) & $51.9(21.6)$ & $64.9(24.1)$ & $<0.05$ \\
Physical role limitations (SF-36) & $36.1(39.4)$ & $50.0(37.6)$ & NS \\
Emotional role limitations (SF-36) & $60.7(44.3)$ & $74.7(36.9)$ & NS \\
Social functioning (SF-36) & $58.6(7.3)$ & $55.7(8.7)$ & NS \\
Mental health (SF-36) & $62.6(21.9)$ & $74.7(18.6)$ & $<0.05$ \\
General health perceptions (SF-36) & $39.3(14.7)$ & $53.2(19.3)$ & $<0.001$ \\
Vitality (SF-36) & $41.9(22.4)$ & $49.8(21.7)$ & NS \\
Pain (SF-36) & $74.0(19.0)$ & $82.2(16.9)$ & NS \\
Sleep (MOS) & $22.4(7.7)$ & $24.2(5.3)$ & NS \\
Anxiety (HAD) & $9.03(4.1)$ & $7.05(3.3)$ & $<0.05$ \\
Depression (HAD) & $6.25(4.4)$ & $3.96(3.6)$ & $<0.05$ \\
\hline
\end{tabular}

NS, not significant. adjusted to the condition had more limited physical function $(p<0.05)$, perceived their health to be less good $(\mathrm{p}<0.001)$, and had more impaired psychological health in terms of mental health, anxiety, and depression scores $(\mathrm{p}<0.05)$ than did those reporting good adjustment. It appears that adjustment to cardiomyopathy is particularly associated with functional status and mental health, rather than with other aspects of quality of life.

\section{MULTIVARIATE PREDICTORS OF QUALITY OF LIFE} AND WELLBEING

Multiple regression analysis was carried out on each measure of quality of life and psychological wellbeing, in order to determine the extent to which scores were predicted by demographic and clinical factors. Exercise capacity was excluded since it showed no univariate associations with quality of life. The results are summarised in table 6 as model 1 . It is evident that demographic and clinical factors made substantial contributions to ratings of vitality, physical functioning, sleep, pain, mental health, and general health perception scales, accounting for $19.4-40.7 \%$ of variance. Demographic and clinical variables did not predict anxiety, depression, role functioning, or social activity.

The value of assessing adjustment to cardiomyopathy was tested by adding this variable in a second step into the regression models. As shown in table 6 , adjustment scores significantly accounted for additional variance in seven of the 11 domains of quality of life and psychological wellbeing. The additional variance accounted for ranged from $6.3 \%$ for social 
Table 6 prediction of quality of life and psychological well being by demographic and clinical factors and by psychological adjustment scores

\begin{tabular}{lllll}
\hline & $\begin{array}{l}\text { Variance } \\
\text { accounted for } \\
\text { by model 1 (\%) }\end{array}$ & Significance & $\begin{array}{l}\text { Variance accounted } \\
\text { for by model 1 + } \\
\text { adjustment (\%) }\end{array}$ & $\begin{array}{l}\text { Significance } \\
\text { F change }\end{array}$ \\
\hline Vitality (SF-36) & 40.7 & $\mathrm{p}<0.001$ & 44.5 & $\mathrm{NS}$ \\
Physical functioning (SF-36) & 39.2 & $\mathrm{p}<0.001$ & 46.3 & $\mathrm{p}<0.05$ \\
Sleep (MOS) & 28.9 & $\mathrm{p}<0.01$ & 29.1 & $\mathrm{NS}$ \\
General health perceptions (SF-36) & 25.2 & $\mathrm{p}<0.025$ & 47.6 & $\mathrm{p}<0.001$ \\
Pain (SF-36) & 21.0 & $\mathrm{p}<0.05$ & 32.6 & $\mathrm{p}<0.01$ \\
Mental health (SF-36) & 19.4 & $\mathrm{p}<0.05$ & 33.8 & $\mathrm{p}<0.01$ \\
Depression (HAD) & 10.1 & $\mathrm{NS}$ & 25.4 & $\mathrm{p}<0.005$ \\
Anxiety (HAD) & 9.4 & $\mathrm{NS}$ & 26.5 & $\mathrm{NS}$ \\
Physical role limitations (SF-36) & 0.04 & $\mathrm{NS}$ & 0.09 & $\mathrm{NS}$ \\
Emotional role limitations (SF-36) & 0.08 & $\mathrm{NS}$ & 3.0 & $\mathrm{p}<0.05$ \\
Social functioning (SF-36) & 0.1 & 6.4 & \\
\hline
\end{tabular}

Model 1 includes age, sex, duration of illness, shortening fraction, left ventricular end diastolic diameter, family history, heart failure, presence of arrhythmia, and mitral regurgitation.

functioning to $22.4 \%$ for general health perceptions. Therefore, several aspects of impaired quality of life in patients with dilated cardiomyopathy are not simple functions of disease severity, but are determined by factors related to psychological adjustment.

\section{Discussion}

This study used standard measures of quality of life and psychological wellbeing to assess the experience of patients with dilated cardiomyopathy. The advantages of this approach are that psychometrically sound and validated measures can be administered, and that scores can be compared with those obtained with other samples and different diseases. The limitation is that standard instruments may not capture all the phenomena associated with cardiomyopathy, so that disease specific measures may be required. ${ }^{15} 16$

Existing literature on quality of life and wellbeing in dilated cardiomyopathy is sparse. Patients with this condition have been included in studies of quality of life in heart failure and atrial tachyarrhythmia, ${ }^{17}$ but often without being identified as a separate group. ${ }^{18}{ }^{19}$ Our results show that patients with dilated cardiomyopathy experience poor quality of life in many domains, including physical function or activities of daily living, emotional and social functioning, vitality, general perceptions of health, and sleep. These limitations are similar in extent to those that we previously found in a sample of patients with hypertrophic cardiomyopathy recruited from the same clinic. ${ }^{3}$ Dilated patients, however, reported greater restrictions in social functioning (adjusted for age and sex), while hypertrophic patients experienced more pain. These restrictions in quality of life are not unique to cardiomyopathy, but are broadly comparable with those experienced by patients with other serious cardiac conditions. The comparison with scores derived from the group of mixed cardiac patients in MOS showed no differences in five of the eight SF-36 scales. Nonetheless, the dilated patients had significantly lower social functioning and poorer mental health scores than the MOS sample.

Dilated patients also report higher levels of anxiety and depression than those prevalent in the population. The raised depression level is similar to that found in coronary artery disease, ${ }^{20}$ but anxiety levels are higher than those found in other cardiac conditions. In combination, our data suggest that patients with dilated cardiomyopathy suffer particularly from anxiety and restrictions in social functioning, in comparison with other cardiac patients.

A possible explanation of heightened anxiety and disturbance is the familial nature of some cases of dilated cardiomyopathy. We anticipated that patients with familial cardiomyopathy might experience greater psychological distress, since the knowledge that a disease can be inherited or passed on to offspring may have emotional consequences. ${ }^{21}$ We found differences in quality of life associated with familial disease, but not as expected (table 4). Patients with familial disease had better physical function, perceived fewer role limitations related to emotional problems, and reported less disturbed sleep than the remainder. The explanation is not clear, but it does not support the notion that knowledge of a familial element has adverse psychosocial effects. One reason for this finding may be that non-familial patients were referred because of their own symptoms, while some individuals in the familial group were only identified and diagnosed through screening of relatives of a proband.

A second aim of this study was to assess associations between clinical features of dilated cardiomyopathy and quality of life and wellbeing. It could be argued that the limitations in quality of life and emotional distress are direct consequences of disease severity and related restrictions of function, with the worst quality of life in patients with the most serious disease. Our data indicate that several aspects of disease are indeed related to poor quality of life; associations with systolic and diastolic myocardial function, heart failure, and mitral regurgitation were all observed (tables 3 and 4). Different aspects of quality of life were affected by different parameters of disease severity. For example, physical functioning was impaired in patients with heart failure, lower left ventricular shortening fractions, and higher left ventricular end diastolic diameters, but was unrelated to the presence of mitral regurgitation or cardiac arrhythmia. Poor social functioning was not related to myocardial function or heart failure, but was greater among patients with moderate or severe mitral regurgitation. Hence, no simple associations between disease 
severity and quality of life and wellbeing could be identified.

It is also apparent that the combined measures of clinical status and disease severity accounted for significant variance in only some aspects of quality of life. As can be seen in table 6 , self reported vitality, physical functioning, sleep disturbance, and perception of health were strongly related to demographic and clinical features. On the other hand, the variance in emotional distress (depression and anxiety) and perceived limitations in roles resulting from disease were not dependent on demographic and clinical features. This implies that other factors are responsible for the variation recorded on some domains of quality of life. Of course, emotional state and role functioning are affected by numerous aspects of life apart from the presence of a specific illness. We found, however, that one important element is the level of psychological adjustment to cardiomyopathy. We have previously shown that poor adjustment to hypertrophic cardiomyopathy is associated with restrictions in social functioning and role performance. ${ }^{3}$ In the present analysis, poor adjustment was unrelated to these aspects of quality of life, but did predict poor physical function, mental health, and emotional distress. Moreover, in multivariate analysis, the adjustment scale increased the variance accounted for in seven of the 11 scales assessed, even after demographic and clinical features had been taken into account (table 6). This finding suggests that the adjustment scale may prove useful as a short screening instrument to identify patients whose impairment in quality of life and emotional distress is greater than would be anticipated on the basis of cardiological measures. Psychological adjustment to cardiomyopathy may depend on effective communication with clinical staff, and psychosocial factors such as support and understanding from family and friends.

This survey was cross sectional in nature, and involved patients who had typically been diagnosed for several years. We do not know, therefore, how deterioration in quality of life evolved with the disease and its identification. The response rate in this study was disappointing $(60.6 \%)$, leaving open the possibility that the patients who responded were not entirely representative. This survey was conducted among patients referred to a specialist unit where particular efforts are made to communicate risk information and alleviate concerns.
The results suggest that despite these efforts, the quality of life of patients with dilated cardiomyopathy is poor, and that these limitations cannot be attributed solely to symptoms or severity of the underlying disease.

1 Dec GW, Fuster V. Idiopathic dilated cardiomyopathy. $N$ Engl f Med 1994;331:1564-75.

2 Richardson P, McKenna W, Bristow M, et al. Report of the 1995 World Health Organization/International Society and Federation of Cardiology task force on the definition and classification of cardiomyopathies. Circulation 1996;93: 841-2.

3 Cox S, O'Donoghue A, McKenna WJ, et al. Health related quality of life and psychological wellbeing in patients with hypertrophic cardiomyopathy. Heart 1997;78:182-7.

4 Poole-Wilson PA. Dilated and restrictive cardiomyopathy. In: Julian DG, Camm AJ, Fox KM, et al, eds. Diseases of the heart, 2nd ed. London: WB Saunders, 1996:482-8.

5 Ware JE Jr, Sherbourne CD. The MOS 36-item short-form health survey (SF-36). I. Conceptual framework and item selection. Med Care 1992;30:473-83.

6 Lyons RA, Lo SV, Littlepage B. Comparative health status of patients with 11 common illnesses in Wales. F Epid Comm Health 1994;48:388-90.

7 Jenkinson C, Wright L, Coulter A. Short form 36 (SF-36) health survey questionnaire: normative data for adults of working age. BMf 1993;306:1437-40.

8 Jenkinson C, Layte R, Wright L, et al. The UK SF-36: an analysis and interpretation manual. Oxford: Health Services Research Unit, 1996

9 Hays RD, Stewart AL. Sleep measures. In: Stewart AL, Ware JE, ed. Measuring functioning and well-being. Durham, North Carolina: Duke University Press, 1992.

10 Zigmond S, Snaith R. The hospital anxiety and depression scale. Acta Psychiatr Scand 1983;86:1-7.

11 Herrmann C. International experiences with the hospital anxiety and depression scale - a review of validation data and clinical results. F Psychosom Res 1997;42:17-41.

12 McHorney CA, Ware JE Jr, Raczek AE. The MOS 36-item short-form health survey (SF-36): II. Psychometric and clinical tests of validity in measuring physical and mental health constructs. Med Care 1993;31:247-63.

13 Lisspers J, Nygren A, Soderman E. Hospital anxiety and depression scale (HAD): some psychometric data for a depression scale (HAD): some psychometric data

14 Herrmann C, Brand-Driehorst S, Kaminsyk B, et al. Diagnostic groups and depressed mood as predictors of 22 -month mortality in medical inpatients. Psychosom Med 1998;60:570-7

15 Guyatt GH, Bombardier C, Tugwell PX. Measuring disease-specific quality of life in clinical trials. Can Med Assoc F 1986;134:889-5.

16 Wiklund I, Waagstein F, Swedberg K, et al. Quality of life on treatment with metoprolol in dilated cardiomyopathy: results from the MDC trial. Cardiovasc Drugs Ther 1996;10:361-8.

17 Tyni-Lenne R, Gordon A, Jansson E, et al. Skeletal muscle endurance training improves peripheral oxidative capacity, exercise tolerance, and health-related quality of life in women with chronic congestive heart failure secondary to either ischemic cardiomyopathy or idiopathic dilated cardiomyopathy. Am f Cardiol 1997;80:1025-9.

18 Fowler MB. Effects of beta blockers on symptoms and functional capacity in heart failure. Am F Cardiol 1997;80:55LL

19 Manolis AG, Katsivas AG, Lazaris EE, et al. Ventricular performance and quality of life in patients who underwent radiofrequency AV junction ablation and permanent pacemaker implantation due to medically refractory atrial tachyarrhythmias. Fournal of Interventional Cardiac Electrophysiology 1998;2:71-6.

20 Musselman DL, Evans DL, Nemeroof CB. The relationship of depression to cardiovascular disease. Arch Gen Psychiatr 1998;55:580-92.

21 Michie S, Marteau T. Genetic counselling: Some issues of theory and practice. In: Marteau T, Richards M, eds. The
troubled helix. Cambridge: Cambridge University Press, 1996:104-22. 